\title{
Contribution of Scanner in the Breast Cancer Extension Assessment in the Radiology Department of CNHU-HKM in Benin
}

\author{
Yekpe Ahouansou Patricia ${ }^{1, ~ *}$, Adjadohoun Sonia ${ }^{1}$, Lokossou Symphorose ${ }^{2}$, Ngamo Gabriel ${ }^{1}$, \\ Lokossou Andreas ${ }^{1}$, Kiki Miralda ${ }^{3}$, Akanni Djivèdé ${ }^{3}$, Biaou Olivier ${ }^{1}$ \\ ${ }^{1}$ Department of Radiology and Medical Imaging, National Teaching University Hospital Hubert Koutoukou Maga (CNHU-HKM), Cotonou, \\ Benin \\ ${ }^{2}$ University Clinic of Gynecology, National Teaching University Hospital Hubert Koutoukou Maga (CNHU-HKM), Cotonou, Benin \\ ${ }^{3}$ Radiology Department of Parakou, Teaching University Hospital of Borgou Department, Parakou, Benin
}

Email address:

Yfrida_pat@yahoo.fr (Y. A. Patricia)

${ }^{*}$ Corresponding author

\section{To cite this article:}

Yekpe Ahouansou Patricia, Adjadohoun Sonia, Lokossou Symphorose, Ngamo Gabriel, Lokossou Andreas, Kiki Miralda, Akanni Djivèdé, Biaou Olivier. Contribution of Scanner in the Breast Cancer Extension Assessment in the Radiology Department of CNHU-HKM in Benin. American Journal of Internal Medicine. Vol. 8, No. 6, 2020, pp. 263-266. doi: 10.11648/j.ajim.20200806.14

Received: October 6, 2020; Accepted: October 27, 2020; Published: November 4, 2020

\begin{abstract}
Objective: Breast cancer is the most common female cancer in the world. The prognosis depends on whether metastasis is present or not. The objective of this study was to provide an overview of the CT scan aspects identified during the evaluation of breast cancer extension in the Radiology department of the Teaching University Hospital of Hubert Koutoukou Maga (CNHU-HKM) in Cotonou in Benin. Materials and methods: It was a descriptive study with retrospective collection, conducted from January 1, 2018 to January 31, 2019 (13 months), in the Radiology department of CNHU-HKM. It focused on patients referred for a chest-abdominal-pelvic computed tomography (PET) scan, as part of an extension assessment for breast cancer. Results: During the period of the study, 40 chest-abdominal-pelvic CT scans were compiled, of which 38 (95\%) were women and 02 men (5\%), and among which 23 were metastatic, accountings for a prevalence of $57.5 \%$. The average age was $51.22 \pm 16.33$ with extremes of 30 and 83 years old. Among our patients, 32 (80\%) had undergone a CT scan for an initial diagnostic of extension assessment, and $08(20 \%)$ for the therapeutic response assessment. According to the TNM classification, T4 tumors accounted for $27.5 \%$ ( 11 cases). Lymph node involvement was found in 18 cases $(43.90 \%)$, accounting for 12 (30\%) of N1 and $6(15 \%)$ of N3. The most commonly found metastatic sites were the lung in $25 \%$ followed by the pleura and the liver with equal percentages (10\%). The metastases CT scan aspect was typically a carcinomatous lymphangitis appearance; fluid pleural effusions; hypodenses, hypovascular nodules hepatics; osteolytic or mixed bone damage and peritoneum, especially fluid effusion associated with peritoneal nodules. Conclusion: The frequency of breast cancer metastases is high in Benin. The most commonly found metastatic sites on scanner in descending order are the lungs, liver and bones.
\end{abstract}

Keywords: Cancer, breast, CT Scan, Metastases, Benin

\section{Introduction}

Breast cancer is the most common female cancer in the world [1].

It is a real public health problem. The prognosis depends on whether metastasis is present or not. The discovery of cancer at the metastasis stage is not uncommon (4-8\%) in Europe [2]. The
CT scan contributes to the extension assessment and conditions the therapeutic attitude. CT is therefore an essential imaging modality for the proper management of metastatic breast cancer. Few studies have been carried out in Benin, on the profile on the scanner during the assessment of breast cancer extension. The objective of this study is to provide an overview of the CT scan aspects identified during the breast cancer extension assessment in the Radiology Department of the Teaching University 
Hospital of Hubert Koutoukou Maga in Benin.

\section{Materials and Methods}

It was a descriptive longitudinal study with retrospective collection, conducted from January 1, 2018 to January 31, 2019 (13 months), in the Radiology department of CNHU-HKM. It focused on patients referred for a chest-abdominal-pelvic computed tomography (PET) scan, as part of an extension assessment for breast cancer. All exams were performed on Siemens Somatom Emotion $®$ (16 CT scanner) model 2011. The patients had a helical acquisition of millimetre axial section with and without intravenous injection of iodized contrast agents followed by multiplanar reconstructions. The interpretations were made by radiologists. The sampling was exhaustive. The source population was patients treated for breast cancer and the target population was patients with metastases.

We included in the study all patient records which the diagnosis of a malignant breast tumour was confirmed by clinical and paraclinical data that underwent CT scan for extension assessment or reassessment. Files with missing or incomplete CT reports were excluded.

The variables studied were socio-demographic (age and sex), clinical (mode of discovery, topography and laterality), and CT scan. The World Health Organization TNM 2012 classification was used.

Data analysis was performed on Epi Info software version 7.0 for the averaging and standard deviation, accepting a statistically significant probability $\mathrm{p}<0.05$. The confidentiality of the files used for the study was respected and the authorizations from the head of the centre and the head of the department were taken.

\section{Results}

\subsection{Epidemiological Data}

During the period of the study, 40 chest-abdominal-pelvic CT scans were collected, including 38 (95\%) women and 02 men $(5 \%)$, out of which 23 were metastatic with a prevalence of $57.5 \%$.

The average age was $51.22 \pm 16.33$ with extremes of 30 and 83 years old.

\subsection{Clinical Data}

\subsubsection{How Metastases Are Discovered}

Among our patients, $32(80 \%)$ had performed a CT scan for an initial diagnostic of extension assessment, 08 (20\%) for an assessment of the therapeutic response.

\subsubsection{Topography of Breast Lesion}

The tumor was either on the right (22 cases accounting for $55 \%$ ) or on the left (17 cases accounting for $42.5 \%$ ). Only one case of bilateral involvement $(2.5 \%)$ was found.

\subsection{CT Scan Data}

\subsubsection{Frequency of Metastases}

A total of 23 patients $(57.5 \%)$ had metastases, including 05 cases of isolated lymph node extension.

These patients were distributed as follows: 18 (56.25\%) out of the 32 patients had performed an initial assessment and 05 $(62.5 \%)$ out of the 08 resorted to therapeutic re-evaluation.

\subsubsection{Metastatic Sites}

Loco-regional extension

Loco-regionally, tumors classified $\mathrm{T} 4$ according to the TNM classification were found in 11 cases $(27.5 \%)$ with an extension to the muscles of the anterior chest wall and ribs.

Lymphatic extension

Lymph node damage was found in 18 cases $(43.90 \%)$ accounting for $12(30 \%)$ of $\mathrm{N} 1$ and $6(15 \%)$ of N3;

Remote Extension

Extra lymphatic locations are shown in Table 1.

Table 1. Distribution of metastatic sites in the population.

\begin{tabular}{lll}
\hline & $\mathbf{N}$ & $\mathbf{\%}$ \\
\hline Lung & 10 & 25 \\
Peura & 4 & 10 \\
Liver & 4 & 10 \\
Peritoneum & 3 & 7.5 \\
Bones & 3 & 7.5 \\
\hline
\end{tabular}

\subsubsection{Metastasis CT Scan Semiology}

The semiology of pulmonary metastases was typically multiple nodules under pleural with a pearly aspect of splinters suggestive of lymphatic distribution (carcinomatous lymphangitis) Pleural involvement was primarily represented by fluid pleural effusions and pleural mass (Figure 1).

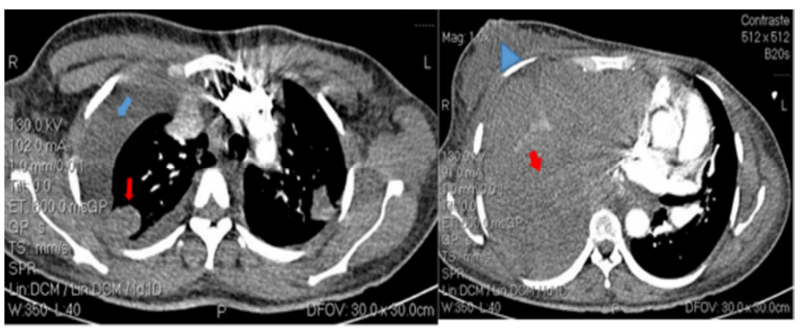

Figure 1. Chest CT imaging Mediastinal window CT scan: pleural fluid effusion (blue arrow), multiple bilateral sub-pleural nodules (red arrows) and right breast masses classified as T4 (blue arrowhead).

Liver lesions were nodules hypodenses hypovascular. (Figure 2).

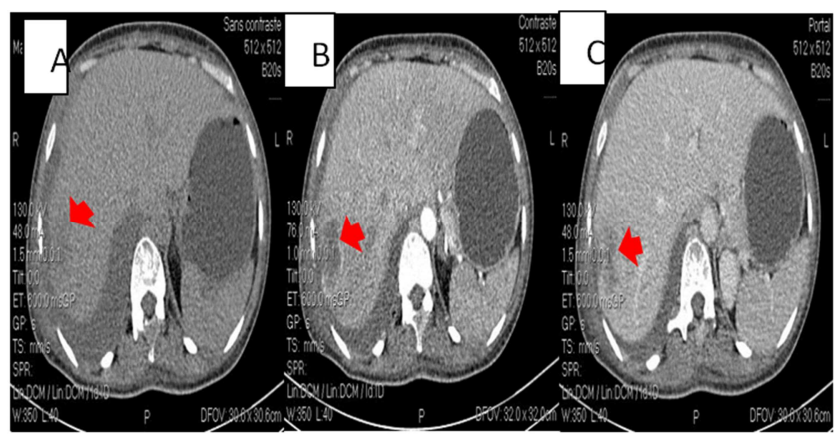

Figure 2. Abdominal axial CT scan sections without injection (A) and with injection of contrast material in the arterial (B) and venous (C) phase: Irregular nodules of segment VII. 
Bone metastases of breast cancer were mostly osteolytic or mixed, rarely osteoecondensant. (Figure 3)

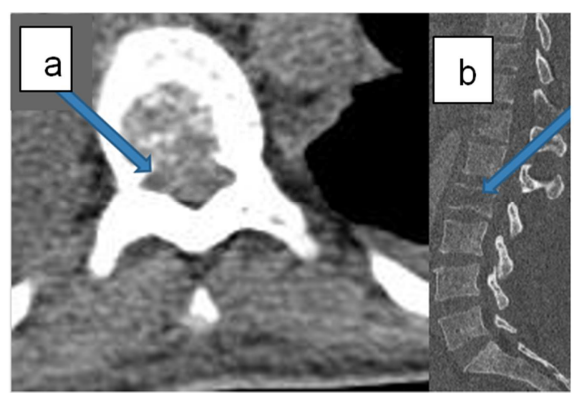

Figure 3. Lumbar spine scan Axial sections after injection of contrast material (a) and sagittal reconstruction (b): 44-year-old patient, osteolytic lesion with compression + epidural tissue mass raised in L2.

Peritoneal lesions were essentially fluid effusions associated with nodules permonial.

\subsection{TNM Classification on the CT}

Table 2 shows the distribution of patients by TNM classification.

Table 2. TNM Classification on CT.

\begin{tabular}{lll}
\hline TNM & $\mathbf{N}$ & $\mathbf{\%}$ \\
\hline T3 & 01 & 2.5 \\
T4 & 11 & 27.5 \\
N1 & 12 & 30 \\
N3 & 06 & 15 \\
M1 & 18 & 35 \\
\hline
\end{tabular}

\section{Discussion}

\subsection{Epidemiological Data}

Breast cancer is the leading female cancer in the world in terms of frequency and the leading cause of death cancer in women with 410.000 cases worldwide. In southern Benin, breast cancer accounts for $65.65 \%$ of gynecological cancers ahead of cervical cancer (19.25\%) and that of the uterine body $(8.10 \%)$ [2]. A key step in managing breast cancer is the imaging work-up and follow-up.

The current recommendations of the European Society for Medical Oncology (ESMO) and the American Society of Clinical Oncology (ASCO) recommend to perform an imaging work-up at the time of diagnosis of breast cancer only in patients with advanced disease: lymph node involvement, tumor size $\geq 5$ $\mathrm{cm}$, clinical signs, anamnesis or biology suggesting the presence of metastases [3, 4]. In our series $80 \%$ had performed an initial diagnostic assessment for extension with $27.5 \% \mathrm{~T} 4,30 \% \mathrm{~N} 1$ and $35 \%$ distant metastasis, which is in line with the recommendations described in the literature. The diagnosis of breast cancer remains late in our series because patients consult at a fairly advanced stage of the disease and the imaging work-up is therefore routinely requested. In 2017 in southern Benin, Lokossou et al published that $53.33 \%$ of patients consulted with a breast tumor higher than $5 \mathrm{~cm}$ [2]. Togo et al in Bamako, Mali, published that $33.81 \%$ of patients consulted at stage IV [5].

The average age of our population was $51.22 \pm 16.33$, similar to that of Lokossou et al [2] in Benin (48 years old), Togo et al [5] in Mali (47.4 years old) and Baeta et al [6] in Lomé (49.5 years old). Almost all clinical studies have shown a linear increase in breast cancer incidence with age [7]. Age is the most well known risk factor for cancer in general and breast cancer in particular. $50 \%$ of breast cancer cases in the US are diagnosed in women over 65 years of age [8]. Only $5 \%$ of our patients were found to be male. Breast cancer in men is an extremely rare pathology representing in Western countries 0.5 to $1 \%$ of breast cancers and 0.4 to $1.2 \%$ of all male cancers [9].

\subsection{Computed Tomography Data}

A total of $23(57.5 \%)$ of the patients had metastases. Lokossou et al [2] found $43.75 \%$ metastasis. However, Brennan et al [10] in a systematic review of 22 publications reported a prevalence ranging from $1.2 \%$ to $34.7 \%$. This difference is due to the fact that in the studies reported by Brennan et al, carried out only in developed countries, the consultation period was early and the breast disease was not that advanced.

Loco-regionally, tumors classified as T4 according to the TNM classification were the most frequently found in $27.5 \%$. JEDIDI JIHEN et al [7] had targeted $47.8 \%$ of T2 tumors in their series. Schroeder et al [11], in a study on the value of extensional imaging assessment at initial diagnosis and follow-up of breast cancer, noted a predominance of $\mathrm{T} 2$ tumors $(42.3 \%)$ followed by T3-4 tumors $(38.5 \%)$. On the other hand, Lokossou et al [2] in South Benin reported 53.33\% $\mathrm{T} 4$ close to our results. The population of Benin seems to be constant in proportions with an advanced stage of local tumor extension.

Lymph node involvements were found in 18 cases $(43.90 \%)$ accounting for $12(30 \%)$ of N1 and $6(15 \%)$ of N3. Schroeder et al [11] also reported more N1 (53.8\%) than N3 (4\%).

We found remote locations affecting, in descending order, the lung $10(25 \%)$, the pleura $4(10 \%)$, the liver $4(10 \%)$, the peritoneum $3(7.5 \%)$ and the bones $3(7.5 \%)$. In the literature the distribution of distant metastatic disease is quite variable. The metastatic sites are identical to our results but diversity in the proportions was noted. Table 3 compares our results with those in the literature. In a meta analysis, Brennan et al [10] reported metastases to bone, liver and lung in descending order, in contrast to our study where lung involvement was predominant. This could be linked to the advanced stage of discovery of the tumors that were locally advanced in our series.

Table 3. Comparison of metastatic sites with those in the literature.

\begin{tabular}{llll}
\hline & Bones (\%) & Liver (\%) & Lung (\%) \\
\hline Our study & 7.5 & 10 & 25 \\
Togo and al [5] & 13.24 & 20.47 & 26.66 \\
Barrett and al [3] & 6.2 & 1.8 & 0.2 \\
Kassm and al [12] & 3.2 & 2.7 & 1.4 \\
Niikura and al [13] & 24.9 & 8.9 & 2.2 \\
\hline
\end{tabular}




\section{Conclusion}

The frequency of breast cancer metastasis is high in Benin. The lack of a systematic screening program and difficult access to care are the cause. The CT made possible to have a precise lesion assessment the most frequent metastatic sites found on CT scan, in descending order are the lungs, liver and bones. The CT semiology of the lesions is identical to that of the literature.

\section{Conflicts of Interest}

The authors declare that they have no competing interest.

\section{References}

[1] Mohammadian M, Pakzad R, Towhidi F, Makhsosi BR, Ahmadi A, Salehiniya H. Incidence and mortality of kidney cancer and its relationship with HDI (Human Development Index) in the world in 2012. Clujul Med. 2017; 90 (3): 286-93.

[2] Mshs L, Ogoudjobi M, Als L, Denakpo J. Cancer mammaire dans le Département du Littoral au Bénin Breast cancer in the Littoral Department of Benin. Carcinol Clin en Afrique. 2017; 16 (2): 26-33.

[3] Barrett T, Bowden DJ, Greenberg DC, Brown CH, Wishart GC, Britton PD. Radiological staging in breast cancer: Which asymptomatic patients to image and how. Br J Cancer. 2009; 101 (9): 1522-8. Available from: http://dx.doi.org/10.1038/sj.bjc.6605323.

[4] Senkus E, Kyriakides S, Penault-Llorca F, Poortmans P, Thompson A, Zackrisson S, et al. Primary breast cancer: ESMO clinical practice guidelines for diagnosis, treatment and follow-up. Ann Oncol. 2013; 24 (SUPPL. 6): vi7-vi23.
[5] Togo A, Traoré A, Traoré C, Dembélé BT, Kanté L, Diakité I, et al. Cancer du sein dans deux centres hospitaliers de Bamako (Mali): Aspects diagnostiques et thérapeutiques. J Africain du Cancer. 2010; 2 (2): 88-91.

[6] Baeta S, Nyame A, Nyame A, Tete K, Kolani K, Akpadza K. Survie globale des patientes traitees pour cancer du sein au Chu de Lome (Togo). J la Rech Sci l’Université Lomé. 2010; 7-8.

[7] Jihen J, Habib A, Nabil T, Sourour Y, Slim C, Khaled T, et al. Le cancer du sein chez la femme agée epidémiologie et caractéristiques cliniques. J. I. M. Sfax. 2004; (19/20) 41-51.

[8] Robert J, Kenn MC. Clinical aspect of cancer in the elderly. Cancer 1994; 74: 2107-2117.

[9] Elbachiri M, Fatima S, Bouchbika Z, Benchekroun N, Jouhadi $\mathrm{H}$, Tawfiq N, et al. Breast cancer in men: About 40 cases and literature review. Pan Afr Med J. 2017; 28: 1-7.

[10] Brennan ME, Houssami N. Evaluation of the evidence on staging imaging for detection of asymptomatic distant metastases in newly diagnosed breast cancer. The Breast. 2012; 21 (2): 112-23. Available from: http://dx.doi.org/10.1016/j.breast.2011.10.005.

[11] Schroeder H, Hanocq, Collignon J, Colin P, DePuiS Z, TriFFaux F, et al. Intérêt du bilan d'extension par imagerie lors du diagnostic initial et du suivi les trois premières années après le diagnostic. Rev Med Liège. 2015; 70 (3): 140-7. Available from: https://orbi.uliege.be/bitstream/2268/181069/1/RMLg 2015 140-147.pdf.

[12] Kasem AR, Desai A, Daniell S, Sinha P. Bone scan and liver ultrasound scan in the preoperative staging for primary breast cancer. The Breast Journal 2006; 12 (6): 544-8.

[13] Niikura N, Costelloe CM, Madewell JE, Hayashi N, Yu TK, Liu J, et al. FDG-PET/CT Compared with conventional imaging in the Detection of distant metastases of primary breast cancer. The Oncologist 2011; 16 (8): 1111-9. 